\title{
Photonic Bands and Gap Maps in a Photonic Crystal Slab
}

\author{
Lucio Claudio Andreani and Mario Agio
}

\begin{abstract}
The photonic bands of a two-dimensional (2-D) lattice patterned in a planar waveguide are calculated by expanding the magnetic field on the basis of waveguide modes. The method yields both the truly guided modes of the structure as well as the quasi-guided modes (or guided resonances) which lie above the light line in the first Brillouin zone. Representative results for the photonic bands are shown in the cases of strong- and weak-confinement waveguides patterned with a triangular lattice of holes. The gap maps as a function of hole radius are calculated and show significant differences with respect to the ideal 2-D case. A comparison of the photonic bands with those extracted from the calculated surface reflectance shows very good agreement, thereby indicating the reliability of the approach.
\end{abstract}

Index Terms-Modeling, optical materials, waveguides.

\section{INTRODUCTION}

$\mathbf{T}$ WO-DIMENSIONAL (2-D) photonic crystals embedded in a planar waveguide, also known as photonic crystal slabs, are being widely investigated [1]-[27] as they can be fabricated at optical wavelengths and may allow control of in-plane light propagation to be achieved. These structures support two kinds of modes. If the waveguide thickness is not too small, guided modes exist whose energies lie below the light line of the cladding material (or light lines, if the waveguide is asymmetric). These modes are true stationary Bloch states and are not subject to scattering losses in an ideal structure without roughness. Above the light line of the cladding material, there exist quasi-guided modes, or guided resonances, which lie within the continuum of leaky modes of the waveguide and therefore have intrinsic radiation losses related to out-of-plane diffraction.

The dispersion of guided modes has first been calculated introducing a supercell in the vertical direction and using a threedimensional (3-D) plane-wave expansion [6], [19]. Both guided and quasi-guided modes can be obtained from the position of resonances in the transmission spectra calculated by the finite difference time-domain (FDTD) method [15], [25]. Recently, a nearly-free photon approximation which starts from the guided modes of the slab taken with a homogeneous refractive index was formulated by Ochiai and Sakoda [26]; the effect of the nonhomogeneous components of the dielectric tensor is included within degenerate perturbation theory.

Manuscript received August 28, 2001; revised March 1, 2002. This work was supported in part by MURST through PRIN 2000 Project "One- and two-dimensional photonic crystals: Growth, theory and optical properties."

The authors are with the INFM and Dipartimento di Fisica "A. Volta," Università di Pavia, I-27100 Pavia, Italy (e-mail: andreani@ fisicavolta.unipv.it).

Publisher Item Identifier S 0018-9197(02)05712-3.
In the present work, the energies of photonic modes in a deeply patterned waveguide are calculated by expanding the magnetic field on the basis of guided modes, where each layer of the waveguide is taken to have an average dielectric constant. This method goes beyond the nearly free approximation of [26], since no perturbative approximation is made and the method is valid even for a strong modulation of the dielectric constant. The guided modes of the "effective" waveguide are folded in the first Brillouin zone and coupled by the inverse dielectric tensor, just like plane waves in ideal 2-D photonic crystals. The eigenmodes which fall below (above) the light line are identified with guided (quasi-guided) modes, respectively, and the energy dispersion of both kinds of modes is obtained at the same time.

In Section II, we give a short outline of the method, including some symmetry aspects. In Section III, we discuss representative results for the photonic bands of two kinds of photonic crystal slabs: the air bridge (a free-standing layer with a large dielectric constant) and the GaAs-AlGaAs system (where the dielectric contrast between the core and cladding is small and all layers are patterned). We focus on the triangular lattice of holes, which is the 2-D structure of the utmost technological interest. In Section IV, the gap maps as a function of hole radius are calculated and compared with the ideal 2-D case. In Section V, we test the soundness of the method by comparing the photonic bands obtained by expansion in waveguide modes with those extracted from surface reflectance calculated by the scattering matrix method [10]. Section VI contains a summary of the results.

\section{METHOD}

Let us consider the second-order equation for the magnetic field

$$
\nabla \times \frac{1}{\epsilon(\mathbf{r})} \nabla \times \mathbf{H}=\frac{\omega^{2}}{c^{2}} \mathbf{H}
$$

where $\epsilon(\mathbf{r})$ is the spatially dependent dielectric constant. If the magnetic field is expanded in an orthonormal set of basis states labeled by the index $\mu$ as

$$
\mathbf{H}(\mathbf{r})=\sum_{\mu} c_{\mu} \mathbf{H}_{\mu}(\mathbf{r})
$$

then (1) is transformed into a linear eigenvalue problem

$$
\sum_{\nu} \mathcal{H}_{\mu \nu} c_{\nu}=\frac{\omega^{2}}{c^{2}} c_{\mu}
$$


where the "Hamiltonian" matrix $\mathcal{H}_{\mu \nu}$ is given by

$$
\mathcal{H}_{\mu \nu}=\int \frac{1}{\epsilon(\mathbf{r})}\left(\nabla \times \mathbf{H}_{\mu}^{*}(\mathbf{r})\right) \cdot\left(\nabla \times \mathbf{H}_{\nu}(\mathbf{r})\right) \mathrm{d} \mathbf{r} .
$$

In the present case of a photonic crystal slab, we have a waveguide along $z$ and a periodic 2-D patterning in the $x y$ plane. The basis set $\mathbf{H}_{\mu}(\mathbf{r})$ is chosen to consist of the guided modes of an effective waveguide, where each layer $j$ is taken to have a homogeneous dielectric constant given by the spatial average of $\epsilon_{j}(x, y)$ within the unit cell [i.e., the $\mathbf{G}=\mathbf{G}^{\prime}$ Fourier component of the dielectric matrix $\left.\epsilon_{j}\left(\mathbf{G}, \mathbf{G}^{\prime}\right)\right]$. The index $\mu$ of the basis states can be written as $\mu=(\mathbf{k}+\mathbf{G}, \alpha)$, where $\mathbf{k}$ is the Bloch vector in the $x y$ plane, $\mathbf{G}$ is a $2-\mathrm{D}$ reciprocal lattice vector and $\alpha=1,2, \ldots, \alpha_{\max }$ is a discrete index which labels the guided modes at wavevector $\mathbf{k}+\mathbf{G}$. The matrix elements $\mathcal{H}_{\mu \mu^{\prime}} \equiv \mathcal{H}_{\mathbf{k}+\mathbf{G}, \alpha ; \mathbf{k}+\mathbf{G}^{\prime}, \alpha^{\prime}}$ of (4) can be calculated by noting that the $\mathrm{d} x \mathrm{~d} y$ integral in each layer $j$ yields the Fourier transform $\epsilon_{j}^{-1}\left(\mathbf{G}, \mathbf{G}^{\prime}\right)$ of the inverse dielectric function. This is the same quantity that appears in the 2-D case and can be calculated as usual by evaluating $\epsilon_{j}\left(\mathbf{G}, \mathbf{G}^{\prime}\right)$ and inverting the resulting matrix [28]. This procedure, which was also used for metallic gratings [29], [30] and has been given a rigorous mathematical basis [31], yields a fast convergence in the number of reciprocal lattice vectors; moreover, it allows one to compare directly with 2-D photonic structure calculations performed with the same wavevector cutoff.

The guided modes $\mathbf{H}_{\mu}(\mathbf{r})$ of the "effective" waveguide represent an orthonormal set of states, however the basis set is not complete since the leaky modes of the waveguide are not included. When the guided modes are folded in the first Brillouin zone, most (sometimes all) of them fall above the light line, i.e., in the energy region of leaky modes: these quasi-guided modes are found here with zero linewidth, since it is the coupling to leaky modes which gives rise to out-of-plane diffraction and therefore to a finite linewidth. Coupling to leaky modes may also produce a shift of the resonance energies of quasi-guided modes, like in the Fano resonance problem [32]: a comparison with an "exact" reflectance calculation, to be shown in Section V, indicates that such a real energy shift is small under usual circumstances. The present method is therefore suited to calculate the (real) energies of guided and quasi-guided modes: it is conceptually similar to the commonly used plane wave expansion in the 2-D case. Besides the wavevector cutoff, it is also useful to specify a maximum number of guided modes of the effective waveguide; moreover, the present choice of the average dielectric constant in each layer is by no means unique. Both issues are discussed in the next sections in connection with specific examples.

The waveguide modes at wavevector $\mathbf{k}+\mathbf{G}$ can be of the transverse electric (TE) or transverse magnetic (TM) type, where "transverse" refers to a vertical plane containing the wavevector. TE and TM waveguide modes at different wavevectors are coupled to each other by the off-diagonal components of the inverse dielectric tensor, thus both must be included at the same time in the basis set. In the case of a symmetric waveguide, mirror symmetry $\sigma_{x y}$ with respect to a horizontal $x y$ plane bisecting the waveguide is a symmetry operation of the system, and the solutions of Maxwell's equations can be
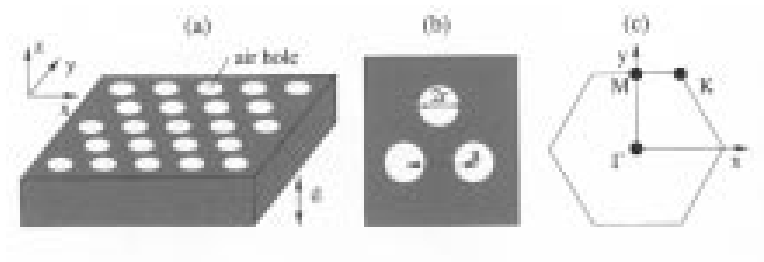

(d)

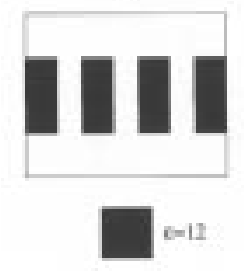

(e)
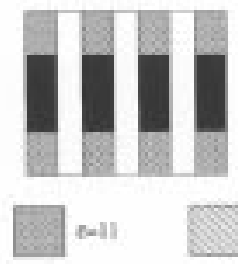

in

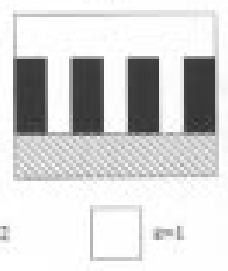

Fig. 1. Upper panels: triangular lattice of circular air holes in a photonic crystal slab. (a) Slab waveguide of thickness $d$ patterned with a triangular lattice. (b) Definition of lattice constant $a$ and hole radius $r$. (c) The 2-D Brillouin zone and symmetry points. Lower panels: side view of the patterned waveguides considered in this work. (d) Strong-confinement symmetric waveguide consisting of a self-standing dielectric slab (air bridge). (e) Weak-confinement symmetric waveguide made of three patterned layers (e.g., AlGaAs-GaAs-AlGaAs system). (f) Asymmetric waveguide consisting of air/patterned core/unpatterned lower cladding (e.g., SOI system). In this paper, we present results for the structures (d) and (e).

classified as even or odd with respect to specular reflection $\sigma_{x y}$. The same symmetry exists in the ideal 2-D case for in-plane propagation, where even solutions are referred to as $H$-modes (nonzero field components $H_{z}, E_{x}, E_{y}$ ) and odd solutions are called $E$-modes (nonzero components $E_{z}, H_{x}, H_{y}$ ). For some special symmetry directions in the Brillouin zone (e.g., the $\Gamma-M$ and $\Gamma-K$ directions in the triangular lattice), a vertical plane $(\hat{k}, \hat{z})$ containing the Bloch vector $\mathbf{k}$ is also a mirror plane of the system: the photonic modes along these symmetry lines may also be classified as even or odd with respect to specular reflection $\sigma_{k z}$. A more general approach which contains all symmetry aspects is the group-theoretical formulation, as performed in [25].

\section{RESULTS FOR PHOTONIC BANDS}

In Fig. 1, we show schematically the triangular lattice of holes in a dielectric slab (a)-(c) and different kinds of patterned waveguides (d)-(f), with the assumed values of the dielectric constant. Fig. 1(d) shows the air bridge, which represents the typical strong-confinement waveguide. Taking $\epsilon=12$ for the dielectric material is appropriate for a $\mathrm{GaAs}$ or $\mathrm{Si}$ membrane. Fig. 1(e) exemplifies a weak-confinement waveguide, which is realized, e.g., in the GaAs-AlGaAs system. In both cases, the waveguide is symmetric. An asymmetric structure is shown in Fig. 1(f), which represents a silicon-on-insulator (SOI) waveguide where only the Si layer is patterned. In all cases, the cladding layers are assumed to be semi-infinite. In this paper, we treat only the structures of Fig. 1(d) and (e). Experimental and theoretical determination of the photonic bands of SOI photonic crystal slabs is reported in [27].

Fig. 2 shows the photonic bands of the air bridge for a hole radius $r / a=0.24$ (air fraction $\sim 0.21$ ) for $d / a=0.3$ and 0.6 , compared with the bands in the ideal 2-D case. These and the 


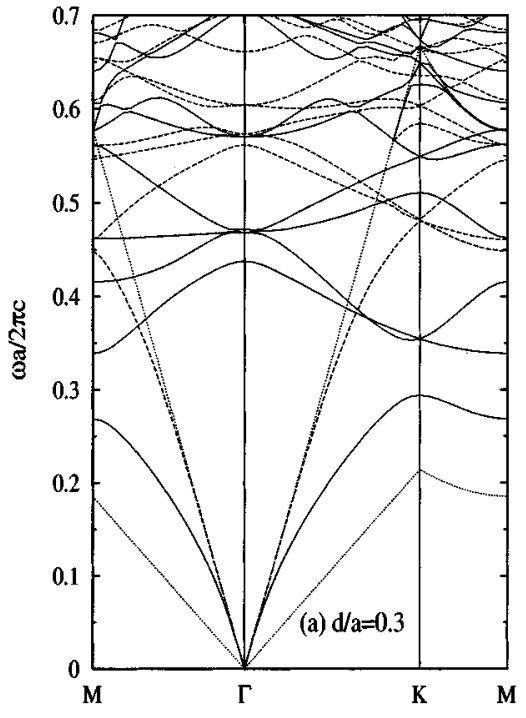

(a)

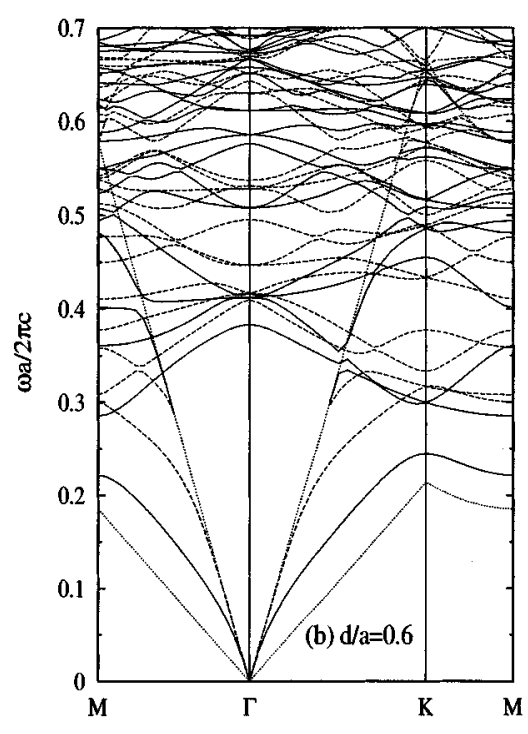

(b)

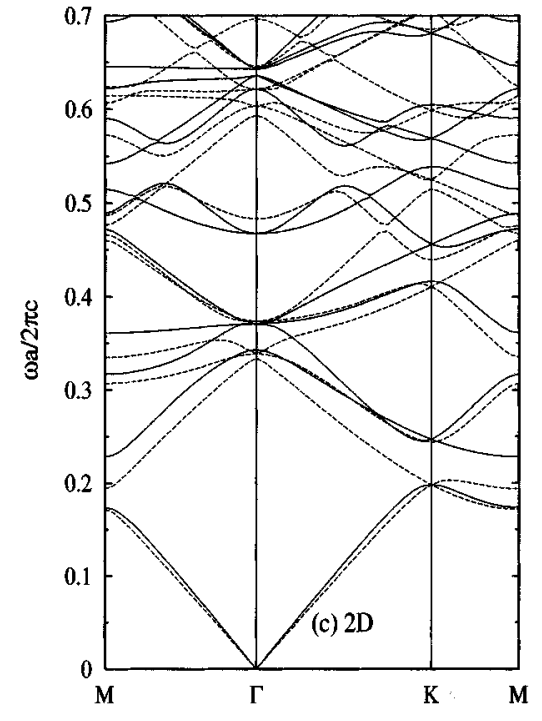

(c)

Fig. 2. Photonic bands for the air bridge structure of Fig. 1(d), for a hole radius $r=0.24 a$ : (a) waveguide thickness $d=0.3 a$; (b) waveguide thickness $d=0.6 a$; and (c) ideal 2-D case. Solid (dashed) lines represent photonic bands which are even (odd) with respect to the horizontal mirror plane $x y$. Dotted lines in (a) and (b) represent the dispersion of light in air and in the effective waveguide material with the average dielectric constant.

following results were obtained using 109 plane waves and up to four guided modes at each wavevector $\mathbf{k}+\mathbf{G}$ for both even and odd states. Convergence in the number of plane waves was carefully checked in the 2-D case, where a much larger cutoff can be used: 109 plane waves are found to give stable photonic bands up to a dimensionless frequency $\omega a /(2 \pi c)=0.7$ over the whole range of hole radii from zero to the close-packing condition $r / a=0.5$. Keeping four guided modes in each parity sector also gives very good convergence, except in the case of thick waveguides with several higher order modes.

The 2-D bands of Fig. 2(c) display well-known features [33]-[37], notably a gap between the first and second bands for even (or $H$ ) modes. The photonic bands of the patterned waveguide fall partly into the guided mode region, where they agree with those calculated in [6], and partly in the leaky mode region above the light cone where they must be viewed as resonances. For $d / a=0.3$ [Fig. 2(a)] the lowest bands are qualitatively similar to their 2-D counterparts, but they are strongly blue-shifted due to confinement in the $z$ direction produced by the waveguide. The gap in the even modes opens between 0.29 and 0.34 [in terms of the dimensionless frequency $\omega a /(2 \pi c)]$, while it lies between 0.2 and 0.23 in the 2-D case. The confinement effect is stronger for odd modes. This is interpreted as follows: the dielectric tensor of the waveguide in the long-wavelength limit is that of a uniaxial medium, with $\epsilon_{\|} \equiv \epsilon_{z z}$ given by the spatial average of the dielectric constant and being larger than $\epsilon_{\perp} \equiv \epsilon_{x x}=\epsilon_{y y}$ [38]. In the 2-D case, odd modes have the electric field along $z$ and feel the largest of the dielectric tensor components; hence, they are better confined in the waveguide and have a larger blue shift compared to even modes. The six photonic modes at the $\Gamma$ point in each polarization can be interpreted as the fundamental waveguide mode at the lowest nonzero reciprocal lattice vectors, which are folded in the first Brillouin zone and split by the dielectric matrix. Note that, in the patterned waveguide with $d / a=0.3$, the photonic modes up to $\omega a /(2 \pi c) \simeq 0.57$ can be put in one-to-one correspondence with the bands of the 2-D case [Fig. 2(c)], thereby indicating that the waveguide is monomode. A second-order waveguide mode appears above $\omega a /(2 \pi c) \simeq 0.57$. Analogous considerations can be made for the case of waveguide thickness $d / a=0.6$ [Fig. 2(b)], where the confinement produced by the waveguide is less pronounced. Moreover, a second-order waveguide mode starts already at $\omega a /(2 \pi c) \simeq 0.3$ and the bands at higher frequencies become more complex.

This example allows us to discuss the trend with respect to waveguide thickness: for a small value of $d / a$, the waveguide is monomode over a wide frequency range and the photonic bands can be interpreted as 2-D bands which are strongly blueshifted, waveguide-induced confinement being stronger for odd modes. When increasing the ratio $d / a$, the blue shift is reduced and a second-order waveguide mode appears with a decreasing cutoff frequency. For $d / a>0.6$ (at a fixed hole radius $r / a=$ 0.24 ), the second-order mode falls into the gap of even modes, thereby contributing to losses when linear defects or cavities are present. In view of achieving guided-wave propagation with the lowest possible losses, it is advisable to employ structures with no quasi-guided modes in the photonic gap; for the air bridge system, small values of $d / a$ are more favorable.

Fig. 3 shows the photonic bands in the weak-confinement situation [structure of Fig. 1(e)] for three different values of the waveguide thickness. Due to the small dielectric contrast between the core and cladding, there are no truly guided modes and all photonic modes lie in the radiative region. The dispersion of quasi-guided modes is very similar to the 2-D case [Fig. 2(c)] and confinement in the waveguide is much less pronounced than for the air bridge. However, it is interesting to observe that the gap in the even modes is increased compared to the 2-D case. The three patterned waveguides are monomode in the whole frequency range shown in the figures, except for $d / a=1$ where a 


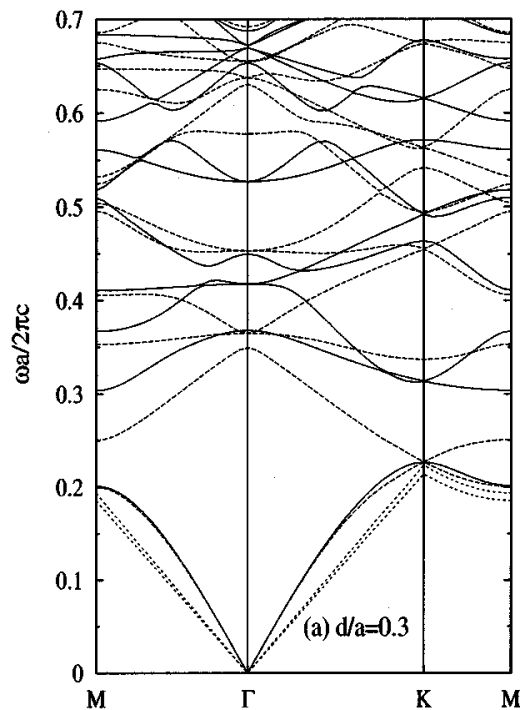

(a)

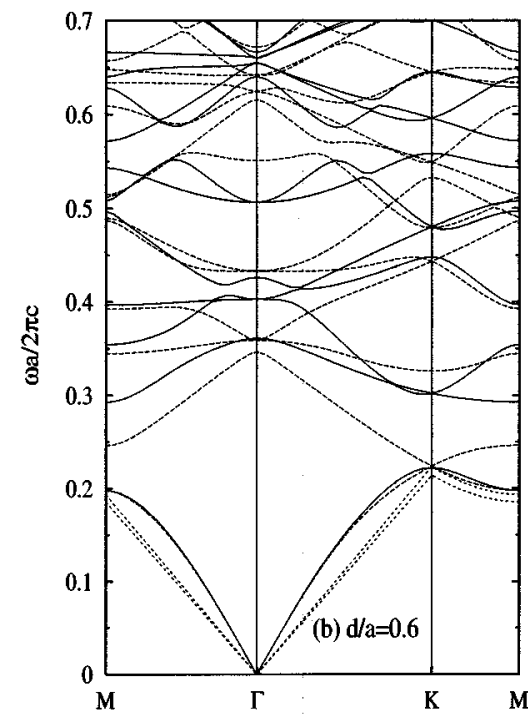

(b)

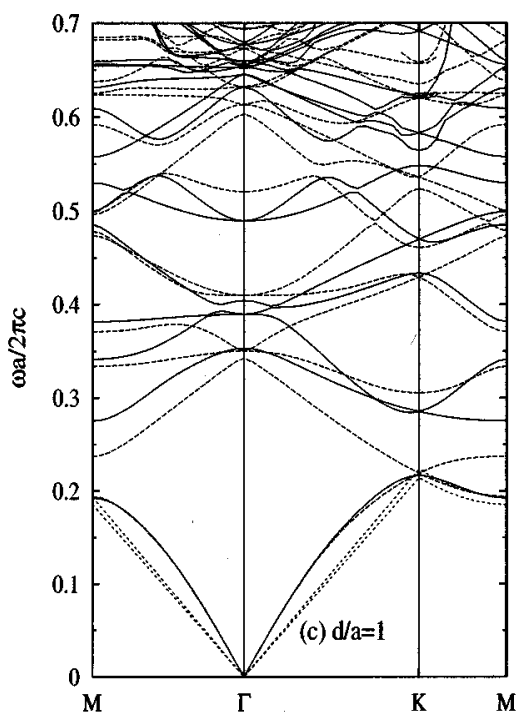

(c)

Fig. 3. Photonic bands for the weak-confinement structure of Fig. 1(e), for a hole radius $r=0.24 a$ : (a) waveguide thickness $d=0.3 a$; (b) $d=0.6 a$; and (c) $d=1.0 \mathrm{a}$. Solid (dashed) lines represent photonic bands which are even (odd) with respect to reflection in the horizontal plane $x y$. Dotted lines represent the dispersion of light in the effective core and cladding materials.

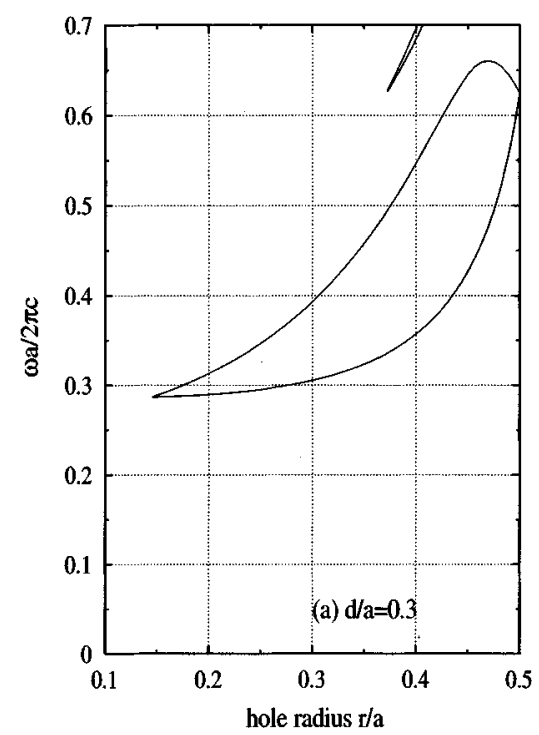

(a)

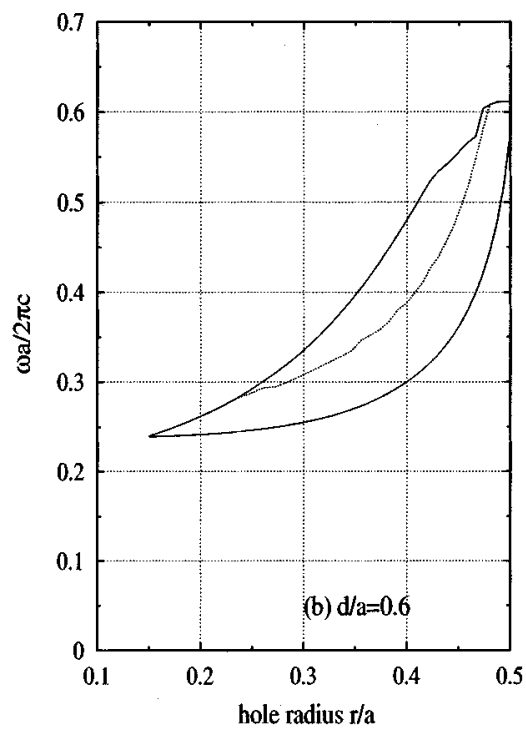

(b)

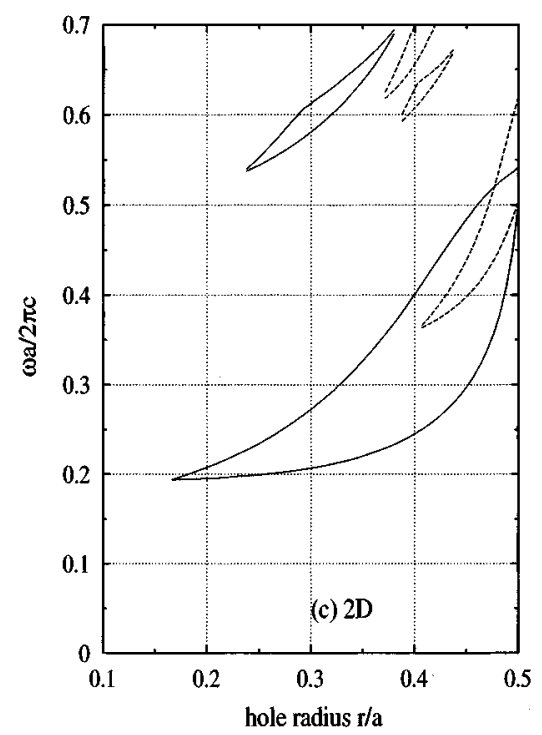

(c)

Fig. 4. Gap maps for the air bridge structure of Fig. 1(d): (a) waveguide thickness $d=0.3 a$; (b) waveguide thickness $d=0.6 a$; and (c) ideal 2-D case. Solid (dashed) lines represent the edges of photonic bands which are even (odd) with respect to specular reflection $\sigma_{x y}$. The dotted line in (b) represents the cutoff of the second-order waveguide mode.

second-order mode appears at $\omega a /(2 \pi c) \simeq 0.65$. Similar to the previous example of the air bridge, the results of Fig. 3 suggest that in order to maximize the even gap it is more convenient to use small values of waveguide thickness.

\section{RESULTS FOR GAP MAPS}

Fig. 4 displays the gap maps as a function of hole radius for the air bridge structure of Fig. 1(d) (waveguide thickness $d / a=$ 0.3 and 0.6) and in the 2-D case. The purpose of Fig. 4(c) is to set a reference for the gap maps in a waveguide and to show that the calculation with 109 plane waves is well converged.
Indeed, the 2-D gap map of Fig. 4(c) agrees with well-known results for the triangular lattice of holes [35]-[37]; in particular, a bandgap common to even and odd modes is present for a hole radius $r / a>0.40$. In the plots of Fig. 4(a) and (b), on the other hand, there is no gap in the odd modes for any hole radius and therefore no complete bandgap. The bandgap for even modes occurs at higher frequencies than in the 2-D case, again due to vertical confinement in the waveguide.

It should be remarked that the upper edge of the gap lies in the radiative region for a hole radius larger than about $0.4 a$ [it can be seen from Fig. 2(a) and (b) that the upper edge is at the K point, where the light line in air has a frequency $\omega a /(2 \pi c) \simeq 0.58$ ]; 


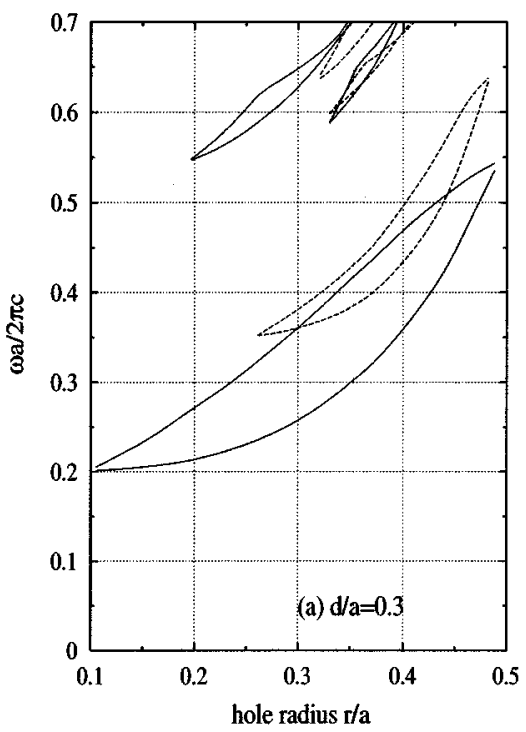

(a)

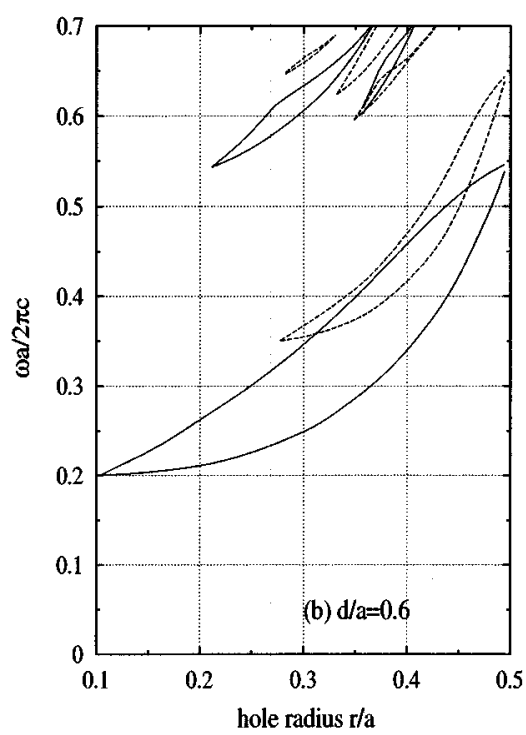

(b)

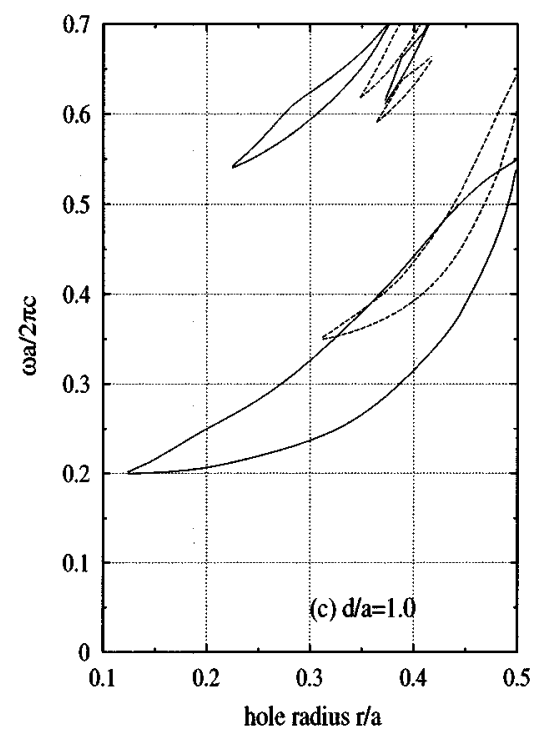

(c)

Fig. 5. Gap maps for the weak-confinement structure of Fig. 1(e): (a) waveguide thickness $d=0.3 a$; (b) $d=0.6 a$; and (c) $d=1.0 a$. Solid (dashed) lines represent the edges of photonic bands which are even (odd) with respect to specular reflection $\sigma_{x y y}$.

thus, the even gap is formed partly in the guided mode region and partly in the radiative region. The bandgaps obtained here are larger than those calculated by Johnson et al. [6], where only the guided mode region was considered. Moreover, in [6], it was concluded that the optimal waveguide thickness for a gap in the even modes is around $d / a=0.6$ and that the bandgap would decrease for smaller thicknesses. By considering both guided and leaky mode regions, we find instead that the even gap of the triangular lattice of holes remains large even for waveguide thicknesses $d / a=0.3$ and below.

The dotted line in Fig. 4(b) represents the cutoff frequency of a second-order waveguide mode [which can be seen in Fig. 2(b)]. Strictly speaking, the even gap exists only between the lower gap edge and the second-order cutoff; for $d / a=1$ (not shown), the second-order cutoff falls below the lower gap edge and there is no even gap altogether. However, it should be remarked that in the present method it is difficult to calculate higher order cutoffs very accurately and that cutoff frequencies may be underestimated. This is due to the choice of the "effective" waveguide for the basis states. Although most features of the photonic band structure (e.g., the gap edges) are rather insensitive to the assumed value of the effective dielectric constant, cutoffs of higher order modes do depend on this parameter. Since the patterned dielectric slab is a uniaxial medium with $\epsilon_{x x}=\epsilon_{y y}<\epsilon_{z z}$, higher order cutoffs calculated considering the dielectric anisotropy will be larger than those estimated using $\epsilon_{z z}$ only. Thus, it is quite possible that the even gap for $d / a=0.6$ is larger than that deduced from the position of the dotted line in Fig. 4(b). In any case, in order to avoid possible complications related to a multimode waveguide, it is more convenient to employ values of $d / a$ smaller than 0.6.

In Fig. 5, we show the gap maps for the weak-confinement waveguide of Fig. 1(e) (waveguide thickness $d / a=0.3,0.6$ and 1). They are rather similar to the 2-D map of Fig. 4(c), again because the confinement effect in the waveguide is much less important than for the air bridge. Two observations must be made. First, the weak-confinement waveguide has no truly guided modes in the considered range of waveguide thicknesses: all photonic modes are resonances in the radiative region and the photonic gap lies entirely in this region. A 2-D photonic gap and defect modes in GaAs-AlGaAs waveguides have been observed and are very promising in view of achieving guided wave propagation [3]-[5], [20], [21]. This lends support to the point of view adopted above, that a bandgap should be defined by the absence of photonic modes in both the guided and radiative regions. Second, on decreasing the waveguide thickness the gap for odd modes opens at smaller values of the hole radius and still overlaps the even gap: a full band gap common to even and odd modes can exist even for hole radii of the order of $0.3 a$, provided waveguides with $d / a \sim 0.3$ are employed.

\section{COMPARISON With Reflectance}

It is known from grating theory (see e.g., the review paper [39]) that the bands of fully periodic waveguides can be derived theoretically by solving the diffraction problem, i.e., by calculating the Bragg reflection spectra to all orders. On the experimental side, photonic bands of deeply patterned waveguides can be measured by the surface coupling technique employed in [8], [9], and [11]: optical reflectance from the surface of the photonic crystal slab displays a series of sharp features with a well-defined dispersion as a function of the incidence angle $\theta$. These resonances correspond to the excitation of photonic modes which are matched in frequency and wavevector to the incoming beam. Each resonant feature marks a point $(\mathbf{k}, \omega)$ of the photonic band dispersion, the wavevector component parallel to the surface being given in modulus by $k=(\omega / c) \sin \theta$. By rotating the sample around its normal, all directions in the 2-D Brillouin zone can be mapped. This technique allows one to probe the photonic bands above the light cone, i.e., in the region of quasi-guided modes. The photonic bands of patterned 


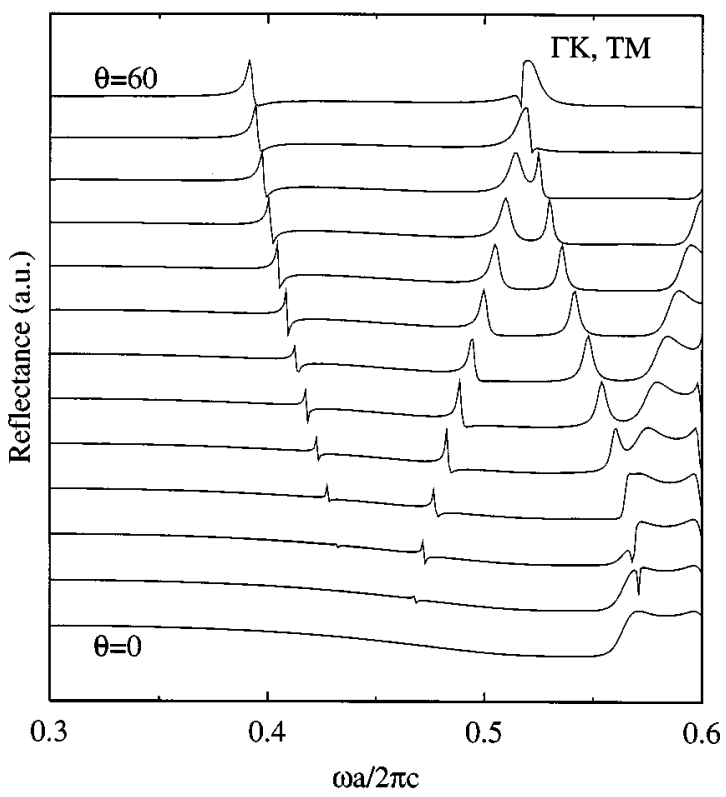

Fig. 6. Calculated surface reflectance of a TM-polarized plane wave incident along the $\Gamma-K$ orientation of the air bridge [waveguide structure of Fig. 1(d)], with thickness $d=0.3 a$ and hole radius $r=0.24 a$. The angle of incidence is varied from $\theta=0^{\circ}$ to $\theta=60^{\circ}$ with a step of $5^{\circ}$.

SOI waveguides have been recently measured by the surface coupling technique [27]. A scattering matrix formalism to calculate the reflectance was developed in [10]; this approach is essentially an exact numerical solution of Maxwell equations (apart from the presence of a wavevector cutoff) for the photonic crystal slab in the presence of a plane wave incident from the surface.

Here we calculate the surface reflectance by the method of [10] and we extract the photonic band dispersion in order to compare with the dispersion calculated by the expansion in waveguide modes. This allows for the assumption of neglecting leaky modes in the expansion to be tested. In Fig. 6, we show the reflectance of a TM-polarized plane wave incident along the $\Gamma-K$ orientation on the surface of an air-bridge photonic crystal slab [Fig. 1(d)], for a hole radius $r=0.24 a$ and a thickness $d=0.3 a$. Sharp resonance features are readily apparent on the reflectance curves and may have the form of maxima, minima, or dispersive lineshapes.

In Fig. 7, we show the photonic band dispersion which is determined from the curves of Fig. 6 (and the analogous ones for other orientations and polarization, not shown) by taking the approximate central position of each resonance. Solid (open) circles in Fig. 7 represent the points extracted from the reflectance curves for TM (TE) polarization with respect to the plane of incidence. They are compared with the photonic bands of the air bridge, which were already given in Fig. 2(a), but for the $\Gamma-M$ and $\Gamma-K$ directions are now classified in terms of parity with respect to specular reflection $\sigma_{k z}$ (see Section II) — the vertical plane $(\hat{k}, \hat{z})$ containing the Bloch vector coincides with the plane of incidence. Modes which are even with respect to $\sigma_{k z}$ (indicated by solid lines) couple only to TM polarized incident light, while odd modes with respect to $\sigma_{k z}$ (dashed lines) couple

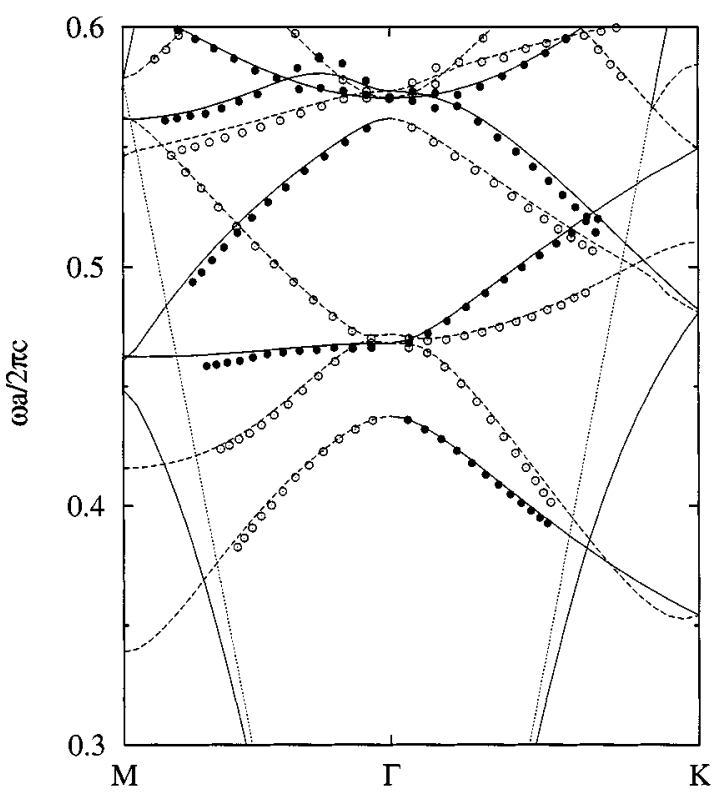

Fig. 7. Photonic bands of the air bridge [waveguide structure of Fig. 1(d)], with thickness $d=0.3 a$ and hole radius $r=0.24 a$. The lines represent the bands calculated from the expansion in waveguide modes, while the points are extracted from the calculated reflectance. Solid lines and closed circles: even modes with respect to a vertical mirror plane $(\mathbf{k}, z)$, probed by TM-polarized light. Dashed lines and open circles: odd modes with respect to a vertical mirror plane $(\mathbf{k}, z)$, probed by TE-polarized light. The dotted line represents the dispersion of light in air.

to TE polarized light. Notice that a linearly polarized plane wave incident from the surface couples to both even and odd modes with respect to $\sigma_{x y}$. There is very good agreement between the photonic bands calculated by the method of Section II and those deduced from reflectance, when the proper parity with respect to $\sigma_{k z}$ is taken into account. This shows that the expansion in waveguide modes of the slab is a reliable method for calculating the energies of quasi-guided modes above the light line, and that the choice of the effective dielectric constant as the spatial average of $\epsilon_{j}(x, y)$ in each layer is appropriate.

Other symmetry aspects of the reflectance calculation and of the photonic bands are worth a comment. First, notice that there are a few bands which are even with respect to $\sigma_{k z}$ along the $\Gamma-M$ direction and become odd along the $\Gamma-K$ direction, or vice versa. They correspond to photonic states which have a three-fold, but not a six-fold, symmetry at the $\Gamma$ point. Second, most resonance features in reflectance become vanishingly small at normal incidence, except for a structure at $\omega a /(2 \pi c) \simeq 0.57$ (see Fig. 6) which remains strong at $\theta=0^{\circ}$ and which splits into two at oblique incidence. Since the only photonic modes which can be excited are those which have the same symmetry of the electromagnetic field, and the latter belongs to a two-fold degenerate representation of the point group at the $\Gamma$ point, nondegenerate bands must be optically forbidden at $\theta=0$. The two-fold degenerate photonic mode at $\omega a /(2 \pi c)=0.57$ is optically allowed and its energy can be determined from normal-incidence reflectance. The same argument implies that nondegenerate bands have zero radiative linewidth at $\mathrm{k}=0$ [25]. 


\section{CONCLUSION}

The photonic bands below and above the light line of photonic crystal slabs can be calculated by expanding the electromagnetic field on the basis of waveguide modes, where each layer of the effective waveguide is defined to have a spatially averaged dielectric constant. The method is conceptually analogous to the usual plane-wave expansion for 2-D systems. Photonic bands and gap maps of strong- and weak-confinement waveguides have been presented and compared to their 2-D counterparts: a photonic gap is defined here by the absence of photonic bands in both the guided and radiative regions. In the strong-confinement (air bridge) structure, photonic bands are strongly blue-shifted with respect to the 2-D case due to confinement in the waveguide, the blue shift being stronger for modes which are odd with respect to the horizontal symmetry plane. The gap maps of the air bridge display only a gap for even modes (not for odd modes). The even gap remains large even for small waveguide thicknesses, while it tends to be eliminated by a second-order waveguide mode for $d / a \sim 0.6$. In the weakconfinement (GaAs-AlGaAs) waveguide, the photonic bands are relatively similar to the 2-D bands; however, the gap maps show quantitative differences. In particular, a complete bandgap opens for smaller values of the hole radius compared to the 2-D case. Comparison of the photonic bands with those extracted from calculated surface reflectance shows good agreement, provided the proper parity with respect to a vertical mirror plane (coinciding with the plane of incidence) is taken into account in the classification of the bands. Thus, the real part of the energy shift due to coupling with leaky modes is not an important effect, at least for the lowest lying bands. The imaginary part of the energy shift is, of course, important, since it describes propagation losses due to out-of-plane diffraction; calculating these losses will require an extension of the present formalism. The expansion in waveguide modes in its present form can also be used to calculate the dispersion of linear defects in photonic crystal slabs within a full three-dimensional treatment.

\section{REFERENCES}

[1] T. F. Krauss, R. M. De La Rue, and S. Brand, "Two-dimensional photonic-bandgap structures operating at near-infrared wavelengths," $\mathrm{Na}$ ture, vol. 383, pp. 699-702, 1996.

[2] S. Fan, P. R. Villeneuve, J. D. Joannopoulos, and E. F. Schubert, "High extraction efficiency of spontaneous emission from slabs of photonic crystals," Phys. Rev. Lett., vol. 78, pp. 3294-3297, 1997.

[3] D. Labilloy, H. Benisty, C. Weisbuch, T. F. Krauss, R. M. De La Rue, V. Bardinal, R. Houdré, U. Oesterle, D. Cassagne, and C. Jouanin, "Quantitative measurement of transmission, reflection, and diffraction of two-dimensional photonic band gap structures at near-infrared wavelengths," Phys. Rev. Lett., vol. 79, pp. 4147-4150, 1997.

[4] D. Labilloy, H. Benisty, C. Weisbuch, C. J. M. Smith, T. F. Krauss, R. Houdré, and U. Oesterle, "Finely resolved transmission spectra and band structure of two-dimensional photonic crystals using emission from InAs quantum dots," Phys. Rev. B, vol. 59, pp. 1649-1652, 1999.

[5] H. Benisty, C. Weisbuch, D. Labilloy, M. Rattier, C. J. M. Smith, T. F. Krauss, R. M. De La Rue, R. Houdré, U. Oesterle, C. Jouanin, and D. Cassagne, "Optical and confinement properties of two-dimensional photonic crystals," J. Lightwave Technol., vol. 17, pp. 2063-2077, 1999.

[6] S. G. Johnson, S. Fan, P. R. Villeneuve, J. D. Joannopoulos, and L. A. Kolodziejski, "Guided modes in photonic crystal slabs," Phys. Rev. B, vol. 60, pp. 5751-5758, 1999.
[7] T. Baba, N. Fukaya, and J. Yonekura, "Light propagation characteristics in defect waveguides in a photonic crystal slabs," Electron. Lett., vol. 35, pp. 654-657, 1999.

[8] V. N. Astratov, D. M. Whittaker, I. S. Culshaw, R. M. Stevenson, M. S. Skolnick, T. F. Krauss, and R. M. De La Rue, "Photonic band-structure effects in the reflectivity of periodically patterned waveguides," Phys. Rev. B, vol. 60, pp. R16255-R16258, 1999.

[9] V. N. Astratov, I. S. Culshaw, R. M. Stevenson, D. M. Whittaker, M. S. Skolnick, T. F. Krauss, and R. M. De La Rue, "Resonant coupling of near-infrared radiation to photonic band structure waveguides," J. Lightwave Technol., vol. 17, pp. 2050-2057, 1999.

[10] D. M. Whittaker and I. S. Culshaw, "Scattering matrix treatment of patterned multilayer photonic structures," Phys. Rev. B, vol. 60, pp. 2610-2618, 1999.

[11] V. N. Astratov, R. M. Stevenson, I. S. Culshaw, D. M. Whittaker, M. S. Skolnick, T. F. Krauss, and R. M. De La Rue, "Heavy photon dispersion in photonic crystal waveguides," Appl. Phys. Lett., vol. 77, pp. 178-180, 2000.

[12] H. Benisty, D. Labilloy, C. Weisbuch, C. J. M. Smith, T. F. Krauss, D. Cassagne, A. Béraud, and C. Jouanin, "Radiation losses of waveguidebased two-dimensional photonic crystals: Positive role of the substrate," Appl. Phys. Lett., vol. 76, pp. 532-534, 2000.

[13] M. Loncar, D. Nedeljkovic, T. Doll, J. Vuckovic, A. Scherer, and T. P. Pearsall, "Waveguides in planar photonic crystals," Appl. Phys. Lett., vol. 77, pp. 1937-1939, 2000.

[14] V. Pacradouni, W. J. Mandeville, A. R. Cowan, P. Paddon, J. F. Young, and S. R. Johnson, "Photonic band structure of dielectric membrane periodically textured in two dimensions," Phys. Rev. B, vol. 62, pp. 4204-4207, 2000.

[15] A. Chutinan and S. Noda, "Waveguides and waveguide bends in two-dimensional photonic crystal slabs," Phys. Rev. B, vol. 62, pp. 4488-4492, 2000.

[16] T. Sondergaard, A. Bjarklev, M. Kristensen, J. Erland, and J. Broeng, "Designing finite-height two-dimensional photonic crystal waveguides," Appl. Phys. Lett., vol. 77, pp. 785-787, 2000.

[17] E. Silvestre, J. M. Pottage, P. St. Russel, and P. J. Roberts, "Design of thin-film photonic crystal waveguides," Appl. Phys. Lett., vol. 77, pp. 942-944, 2000.

[18] E. Chow, S. Y. Lin, S. G. Johnson, P. R. Villeneuve, J. D. Joannopoulos, J. R. Wendt, G. A. Vawter, W. Zubrzycki, H. Hou, and A. Alleman, "Three-dimensional control of light in a two-dimensional photonic crystal slab," Nature, vol. 407, pp. 983-986, 2000.

[19] S. G. Johnson, P. R. Villeneuve, S. Fan, and J. D. Joannopoulos, "Linear waveguides in photonic-crystal slabs," Phys. Rev. B, vol. 62, pp. 8212-8222, 2000.

[20] C. J. M. Smith, H. Benisty, S. Olivier, M. Rattier, C. Weisbuch, T. F. Krauss, R. M. De La Rue, R. Houdré, and U. Oesterle, "Low-channel waveguides with two-dimensional photonic crystal boundaries," Appl. Phys. Lett., vol. 77, pp. 2813-2815, 2000.

[21] S. Olivier, M. Rattier, H. Benisty, C. Weisbuch, C. J. M. Smith, R. M. De La Rue, T. F. Krauss, U. Oesterle, and R. Houdré, "Ministopbands of a one-dimensional system: The channel waveguide in a two-dimensional photonic crystal," Phys. Rev. B., vol. 63, no. 113311, 2001.

[22] Ph. Lalanne and H. Benisty, "Out-of-plane losses of two-dimensional photonic crystal waveguides: Electromagnetic analysis," J. Appl. Phys., vol. 89, pp. 1512-1514, 2001.

[23] N. Kawai, K. Inoue, N. Ikeda, N. Carlsson, Y. Sugimoto, K. Asakawa, S. Yamada, and Y. Katayama, "Transmittance and time-of-flight study of $\mathrm{Al}_{x} \mathrm{Ga}_{1-x}$ As-based photonic crystal waveguides," Phys. Rev. B, vol. 63, no. 153313, 2001.

[24] N. Kawai, K. Inoue, N. Carlsson, N. Ikeda, Y. Sugimoto, K. Asakawa, and T. Takemori, "Confined band gap in an air bridge type of twodimensional AlGaAs photonic crystal," Phys. Rev. Lett., vol. 86, pp. 2289-2292, 2001.

[25] T. Ochiai and K. Sakoda, "Dispersion relation and optical transmittance of a hexagonal photonic crystal slab," Phys. Rev. B, vol. 63, no. 125107, 2001.

[26] — "Nearly free-photon approximation for two-dimensional photonic crystal slabs," Phys. Rev. B, vol. 64, no. 045108, 2001.

[27] M. Patrini, M. Galli, F. Marabelli, M. Agio, L. C. Andreani, D. Peyrade, and Y. Chen, "Photonic bands in patterned silicon-on-insulator waveguides," IEEE J. Quantum Electron., vol. 38, pp. 881-886, July 2002. 
[28] K. M. Ho, C. T. Chan, and C. M. Soukoulis, "Existence of a photonic band gap in periodic dielectric structures," Phys. Rev. Lett., vol. 65, pp. 3152-3155, 1990.

[29] Ph. Lalanne and G. M. Morris, "Highly improved convergence of the coupled wave method for TM polarization," J. Opt. Soc. Amer. A, vol. 13, pp. 779-784, 1996.

[30] S. G. Granet and B. Guizal, "Efficient implementation of the coupled wave method for metallic lamellar gratings in TM polarization," J. Opt. Soc. Amer. A, vol. 13, pp. 1019-1023, 1996.

[31] L. Li, "Use of Fourier series in the analysis of discontinuous periodic structures," J. Opt. Soc. Amer. A, vol. 13, pp. 1870-1876, 1996.

[32] U. Fano, "Effects of configuration interaction on intensities and phase shifts," Phys. Rev., vol. 124, pp. 1866-1878, 1961.

[33] P. R. Villeneuve and M. Piché, "Photonic band gaps in two-dimensional square and hexagonal lattices," Phys. Rev. B, vol. 46, pp. 4969-4972, 1992.

[34] R. D. Meade, K. D. Brommer, A. M. Rappe, and J. D. Joannopoulos, "Existence of a photonic band gap in two dimensions," Appl. Phys. Lett., vol. 61, pp. 495-497, 1992.

[35] J. M. Gérard, A. Izraël, J. Y. Marzin, R. Padjen, and F. R. Ladan, "Photonic bandgap of two-dimensional photonic crystals," Solid-State Electron., vol. 37, pp. 1341-1344, 1994.

[36] R. Padjen, J. M. Gérard, and J. Y. Marzin, "Analysis of the filling pattern dependence of the photonic bandgap for two-dimensional systems," $J$. Mod. Opt., vol. 41, pp. 295-310, 1994.

[37] J. D. Joannopoulos, R. D. Meade, and J. N. Winn, Photonic Crystals. Princeton, NJ: Princeton Univ. Press, 1995.

[38] P. Halevi, A. A. Krokhin, and J. Arriaga, "Photonic crystal optics and homogenization of 2D periodic composites," Phys. Rev. Lett., vol. 82, pp. 719-722, 1999.

[39] E. Popov, "Light diffraction by relief gratings," Progr. Opt., vol. 31, pp. 141-187, 1993.

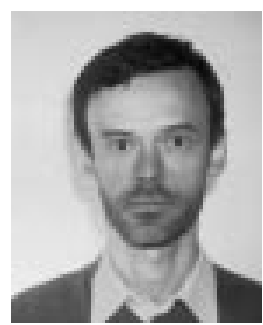

Lucio Claudio Andreani received the Ph.D. degree in physics from Scuola Normale Superiore, Pisa, Italy, in 1989 with a dissertation on excitons and polaritons in semiconductor nanostructures.

He was a Post-Doctoral Fellow at the IRRMA-Ecole Polytechnique Fédérale de Lausanne, Lausanne, Switzerland, working on strongly correlated electron systems. In 1992 he became a Researcher and in 1998 an Associate Professor at the University of Pavia, Pavia, Italy. He is also an Associate with the INFM-National Institute for the Physics of Matter. His research interests span several areas in theoretical condensed matter physics, in particular the optical properties of semiconductors and their heterostructures. His most significant works concern excitons and radiation-matter interaction in quantum wells and in microcavities. Since 2000, he has been involved in the physical investigations of photonic crystals, both from the theoretical side and in collaboration with experimentalists in Pavia and in other laboratories.

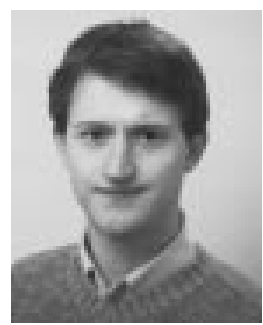

Mario Agio received the Diploma in physics in 1999 from the University of Pavia, Pavia, Italy, where he is currently working toward the Ph.D. degree in physics in conjunction with Iowa State University through the Memorandum of Agreement "International Doctorate."

He was a Research Scholar at Ames Laboratory DOE from September 1999 to December 1999. He is also associatd with the INFM-National Institute for the Physics of Matter and to Ames Laboratory, DOE. His research activity concerns the theory of photonic crystals. He is performing calculations of photonic bands and reflectance, as well as finite- difference time-domain (FDTD) simulations. In particular he is studying the propagation properties of photonic-crystal waveguides and resonators. 